\title{
Discovery of fungal metabolites bergenin, quercitrin and dihydroartemisinin as potential inhibitors against main protease of SARS-CoV-2
}

\author{
Ravi S Patel, Akash G Vanzara, Nimisha R Patel, Ajit M Vasava, Sachin M Patil and \\ Kishore S Rajput* \\ Department of Botany, Faculty of Science, The Maharaja Sayajirao University of Baroda, \\ Vadodara 390002, India. *Corresponding author e-mail: ks.rajput15@yahoo.com
}

\begin{abstract}
Emergence of severe acute respiratory syndrome corona virus 2 (SARS-CoV-2) infection has given rise to COVID-19 pandemic, that is wreaking havoc worldwide. Therefore, there is an urgent need to find out novel drugs to combat SARS-CoV-2 infection. In this backdrop, the present study was aimed to assess potent bioactive compounds from different fungi as potential inhibitors of SARS-CoV-2 main protease $\left(\mathrm{M}^{\mathrm{pro}}\right)$ using an in-silico analysis. Nearly 118 bioactive compounds were extracted from Dictyophora indusiata, Geassstrum triplex and Cyathus stercoreus and identified using HR LC/MS analysis. Of which, only bergenin (D. indusiata), quercitrin (G. triplex) and dihydroartemisinin (C. stercoreus) were selected based on their medicinal uses, binding score and active site covered. The 6LU7, a protein crystallographic structure of SARS-CoV-2 $\mathrm{M}^{\text {pro }}$, was docked with bergenin, quercitrin and dihydroartemisinin using Autodock 4.2 and the binding energies obtained were -7.86, -10.29 and $-7.20 \mathrm{kcal} / \mathrm{mol}$, respectively. Bergenin, quercitrin and dihydroartemisinin formed hydrogen bond, electrostatic interactions and hydrophobic interactions with foremost active site amino acids THR190, GLU166, GLN189, GLY143, HIS163, HIS164, CYS145 and PHE140. Present investigation suggests that these three drugs may be used as alternative inhibitors against SARS-CoV-2 $\mathrm{M}^{\text {pro }}$. However, further research is necessary to assess in vitro potential of these drugs. To the best of our knowledge, present investigation reported these three bioactive compounds of fungal origin for the first time.
\end{abstract}

Keyword: SARS-CoV-2, Bergenin, Quercitrin, Dihydroartemisinin, Fungi, HR LC/MS 


\section{Introduction}

Coronaviruses (CoVs) are positive-sense, enveloped, non-segmented, single-stranded RNA viruses belonging to the Coronaviridae family of the order Nidovirales [1]. CoVs are classified into three main groups based on their antigenic properties: i) alpha-CoVs, ii) beta$\mathrm{CoVs}$, and iii) gamma-CoVs. The alpha-CoVs are responsible for gastrointestinal disorders. The beta-CoVs further divided into (a) Bat coronavirus (BCoV), (b) the human Severe Acute Respiratory Syndrome (SARS) virus, and (c) Middle Eastern Respiratory Syndrome (MERS) virus. The gamma-CoVs, mainly infect avian species [2] while the MERS-CoV and SARS$\mathrm{CoV}$ are the most well-known $\mathrm{CoVs}$ responsible for causing severe respiratory symptoms in Saudi Arabia during 2012 and in southern China during 2002-2003, respectively [3,4]

MERS-CoV and SARS-CoV are thought to have originated in bats and have been transmitted directly to humans from market dromedary camels and civets, respectivelyb [1]. A number of unusual pneumonia cases were reported in Wuhan (China) during December 2019 which were caused by novel SARS-CoV-2, which are now called as Covid-19 [5,6]. The symptoms include fever, common cold, cough, sore throat, nasal congestion, malaise, headache, muscle pain, severe acute respiratory failure [7,8]. In March 2020, the world health organization (WHO) declared SARS-CoV-2 outbreak as a "pandemic" because it has subsequently spread throughout China and in the entire world with the mortality rate of 3\% [9]. To date, 8,602,020 patients have been confirmed to have COVID-19, of which, nearly 456,791 have died [10] and the number is increasing drastically every day.

During SARS-CoV-2 replication, virus proteases play a pivotal role in the assembly of the viral structure that has been suggested crucial for the infection and pathogenicity of SARSCoV-2 [5,9]. Therefore, virus proteases are being considered as a great target for drug discovery [11]. In $\mathrm{CoV}$, the protease $\mathrm{M}^{\text {pro }}$ (main protease) is essential for virus replication, which is encoded by poly protein $1(\mathrm{pp} 1) . \mathrm{M}^{\mathrm{pro}}$ is a 3-chymotrypsin-like cysteine protease $\left(3 \mathrm{CL}^{\text {pro }}\right.$ ) with a molecular weight of $\sim 33.8 \mathrm{kDa}[12,13] . \mathrm{M}^{\text {pro }}$ from SARS-CoV-2 was successfully crystallized and the structural information was deposited in Protein Data Bank (PDB) with accession number 6LU7 [14]. Additionally, $\mathrm{M}^{\mathrm{pro}}$ is being considered as a potential target for inhibition studies as it is highly conserved amongst CoVs [15] and does not have any homology with $\mathrm{M}^{\text {pro }}$ of the human genome $[8,13]$. 
Currently, remarkable and extensive research activities have initiated to counter the SARSCoV-2 infection. Diurnal upgrading with various approaches such as molecular dynamic, docking, drug discovery, in-vivo, in-vitro analysis has the potential to challenge this pandemic. To determine an effective therapeutic regimen for SARS-CoV-2, many trials have been designed at present, of which chloroquine therapy is being considered out of the targeted regimens [16]. Based on Chinese clinical trials, chloroquine phosphate and aminoquinoline could be more effective against SARS-CoV-2 at dose of $500 \mathrm{mg} /$ day which has already used to treat malaria. Hydroxychloroquine is a less toxic aminoquinoline and more soluble than chloroquine because of $\mathrm{N}$-diethyl group of chloroquine has been replaced by an N-hydroxyethyl side chain. It is preferred over chloroquine due to its greater availability in many counties and less adverse side effects [17]. Recently, remdesivir was proven to be effective in the inhibition of SARS-CoV-2 infection using in vitro study [18] as no adverse reactions were observed in the US patients treated with remdesivir [19].

Fungi represent a rich source of several bioactive compounds, of which few possess antiviral activities that can be utilized for drug development against SARS-CoV-2 infection. Various studies have reported antiviral activities of fungal metabolites against HIV, hepatitis $\mathrm{C}$ virus (HCV) and SARS-CoV-2. A paclitaxel, which is now being considered for its inhibitory effect on HIV-1 protease activity, is produced by several fungi like Alternaria, Aspergillus, Cladosporium, Fusarium, Penicillium, Phomopsis, Trichoderma and Xylaria [20]. Similarly, two semi-cochliodinols isolated from Chrysosporium merdarium showed an inhibitory effect on HIV-1 protease activity [21]. Hexane extract obtained from Auricularia polytricha could effectively inhibit HIV-1 protease activity in vitro [22] whereas alternaroil and alternariol extracted from Alternaria alternata have been explored for the inhibition of HCV protease [23]. Furthermore, H1-A isolated from Fusarium oxysporum has displayed activity against $\mathrm{HCV}$ protease [24] and flaviolin, a fungal metabolite is a potent inhibitor of $3 \mathrm{CL}^{\text {pro }}$ of novel corona virus SARS-CoV-2 [25].

As an emergency, the primary motive for all the Covid-19 affected nations is to find efficient therapeutics as there is no apparent approved or clinically tested anti-viral drug for SARSCoV-2. In this context, the present study was focused on finding alternative antiviral drugs against SARS-CoV-2 infection. The present research work investigated three potential 
bioactive compounds, viz. bergenin, dihydroartemisinin and quercitrin as potential inhibitors against main protease of SARS-CoV-2.

\section{Materials and Methodology}

\subsection{Fungal strains}

Fungal strains used in the present study were collected from Jambughoda Wild Life Sanctuary (Panchmahal), Vansda National Park (Dang) and Ratanmahal Wild Life Sanctuary (Dahod), Gujarat state, India. The fungal strains were identified based on morphological characters as well as by the molecular sequencing (rDNA ITS) method as Geassstrum triplex (Accession no. MF506821), Dictyophora indusiata (Accession no. MF510374) and Cyathus stercoreus (Accession no. MH543350). Fruiting bodies of the collected fungal material was dried in the oven and grounded with an electric grinder into a fine powder, followed by storage in an airtight container.

\subsection{Extraction of fungal compounds}

Dry powder $(20 \mathrm{~g})$ of the three fungi were soaked in $85 \%$ ethanol overnight under vigorous shaking condition $\left(110 \mathrm{rpm}\right.$ at $\left.30^{\circ} \mathrm{C}\right)$. The ethanol phase was filtered using Whatman No. 1 filter paper and then concentrated using a rotary evaporator to obtain the dried residue. The obtained residues were further dissolved in $10 \%$ DMSO and used for the profiling of bioactive metabolites.

\subsection{Identification of bioactive components of fungal extracts by TOF/Q-TOF Mass Spectrometer (HR LC/MS)}

Profiling of bioactive compounds from the crude extract of selected fungi was carried out via UHPLC-PDA-Detector Mass Spectrophotometer (HR-LCMS 1290 Infinity UHPLC System), Agilent Technologies ${ }^{\circledR}$, USA. The liquid chromatographic system consisted of MS Q-TOF (G6550A), ion source - Dual AJS ESI, Binary pump (G4220B) HiP sampler (G4226A) and Column Comp. (G1316C). Three microlitres of samples were injected into the system, followed by separation in SB-C18 column $(2.1329 \times 50 \mathrm{~mm}, 1.8 \mu \mathrm{m}$ particle size $)$. The mobile phase used was $100 \%$ water (aqueous formic acid $0.1 \%(\mathrm{v} / \mathrm{v})$ - solvent A) and $100 \%$ acetonitrile $(90 \%$ acetonitrile $+10 \%$ Water $+0.1 \%$ formic acid - solvent B). The initial conditions were $95 \%$ of solvent A and $5 \%$ of solvent B for 2 min, followed by $100 \%$ B in 16 min. This solvent composition was maintained for $8 \min (100 \% \mathrm{~B})$ followed by a return to 
the initial conditions. The flow rate was set to $100 \mu \mathrm{L} / \mathrm{min}$. Compounds were identified using their mass spectra and their unique mass fragmentation patterns.

\subsection{Virtual Screening}

\subsubsection{Proteins/Macromolecules}

Virtual screening was initiated using the crystallographic structure of SARS-CoV-2 $\mathrm{M}^{\text {pro }}$ with an inhibitor N3 [13]. The structure was retrieved from Protein Data Bank (PDB) with accession number 6LU 7 (Deposited: 2020-01-26; Released: 2020-02-05). The 6LU7 protein is a homodimer with two chains, A and C. Macromolecule preparation was carried out using chain A. The native ligand for 6LU7 is N-[(5-METHYLISOXAZOL-3YL)CARBONYL]ALANYL-L-VALYL-N 1 -((1R,2Z)-4-(BENZYLOXY)-4-OXO-1\{[(3R)-2-OXOPYRROLIDIN-3-YL]METHYL\}BUT-2-ENYL)-L-LEUCINAMIDE [26].

\subsubsection{Ligand and drug download}

ZINC and PubChem database were used to obtain 3D structures of natural compounds. Bergenin (ZINC4046820) and quercitrin (ZINC4175638) were downloaded from ZINC database [27] whereas dihydroartemisinin (CID 456410) was downloaded from PubChem [28] in sdf format. Downloaded compounds were restricted to Lipinski's rule of five for drug-likeness properties which include no more than 10 hydrogen bond acceptors, no more than 5 hydrogen bond donors, molecular weights between 160 and $500 \mathrm{~g} / \mathrm{mol}$ and $\operatorname{logP}$ between -0.4 and 5.6 [29]. Drug-likeness properties with Lipinski's rule of five were calculated using SWISSADME prediction [30].

\subsubsection{Determination of active sites}

The co-ordinates of $\mathrm{N} 3$ binding site on SARS-CoV-2 $\mathrm{M}^{\text {pro }}$ were determined using UCSF Chimera (version 1.14), Biovia Discovery Studio 2020 and also according to previous studies with the crystallographic structure of SARS-CoV $-2 \mathrm{M}^{\mathrm{pro}}[9,13,18,31]$. The determined amino acids (THR190, GLU166, GLN189, GLY143, HIS163, HIS164, CYS145 and PHE140) in the active site were used to analyse the grid box for evaluation of docking results.

\subsubsection{Molecular Docking}

Ligand was first converted into pdb format from sdf format using Open Babel GUI 3.0 software. The structures of all the ligand molecules and 6LU7 protein molecule were converted to their corresponding pdbqt files using AutoDock Tools 1.5.6 [32]. Protein 
optimization was carried out by removing water and other atoms, adding polar hydrogen group and Kollman charges using AutoDock version 4.2.6 which is supported by AutoDock tools, MGL tools, AutoDock vina and AutoGrid. The grid coordinates (X, Y, and Z) were arranged on the active site region to define a gridbox. Each docking run was performed 4 times using genetic algorithm (GA) parameters with 1750000 generations. The docking analysis was performed by AutoDock 4.2.6. The 10 best docking hits were selected based on the average of affinity energy values. To confirm molecule interactions with the amino acids within the protease active site, the docking positions were analysed using PyMol 2.3 [33]. Discovery Studio Visualizer 2020 was used for generating 2D maps of receptor-ligand interactions.

\subsubsection{ADMET study}

The predicted Absorption Distribution Metabolism, Excretion and toxicity (ADMET) study were analyzed using the pkCSM - pharmacokinetics server [34]. The sdf file of the compounds were converted into SMILES (Simplified Molecule Input Line Entry Specification) using Open Babel GUI 3.0 software and uploaded to pkCSM to calculate ADMET properties using default parameters.

\section{Results and discussion}

\subsection{Identification of bioactive components of fungal extracts by TOF/Q-TOF Mass Spectrometer (HR LC/MS)}

Fungi are well known for their significant bioactive potentials including anti-viral capability; therefore, crude extract of three different fungi were used for profiling of their bioactive compounds using HR-LCMS. With the detailed mass spectrum data, absorbance spectra and retention times were compared with the available literature and found that all three fungi hold a total of 118 bioactive compounds of different classes. Based on medicinal uses, binding energy and active site covered after molecular docking analysis, three potent compounds (bergenin, quercitrin and dihydroartemisinin) were selected for in depth in-silico analysis as potential inhibitors against SARS-CoV-2 $\mathrm{M}^{\text {pro }}$. 


\subsection{Virtual screening}

The crystal structure of SARS-CoV-2 $\mathrm{M}^{\text {pro }}$ in complex with inhibitor N3 to 2.1- $\AA$ resolution was determined by Jin et al., 2020 [13]. The SARS-CoV-2 M $^{\text {pro }}$ has a Cys-His catalytic dyad. Each protomer of SARS-CoV-2 $\mathrm{M}^{\text {pro }}$ is composed of three domains (Figure 1C). Domains I and Domains II have an antiparallel $\beta$-barrel structure which are extended from residues 8101 and residues 102-184, respectively. Domain III ranges from residues 201-303 and contains five $\alpha$-helices arranged into antiparallel globular cluster. Domain II and Domain III is connected by a long loop region extended from residues 185-200. The substrate-binding site is situated in a cleft between Domain I and II. The inhibitor N3 binds in the substrate binding pocket. The backbone of N3 forms an antiparallel sheet with 164 to168 residues (His164, Met165, Glu166, Leu167, Pro168) of the long strand 155-168 on one side. The backbone of N3 also forms an antiparallel sheet with residues 189-191 (Gln189, Thr190, Ala191) of the loop linking domain II and III. The inhibitor N3 makes van der Waals bond, conventional hydrogen bond, carbon hydrogen bond, amide $\pi$-stacked interaction, alkyl and $\pi$-alkyl interactions with different residues of SARS-CoV-2 $\mathrm{M}^{\text {pro }}$ (Figure 1D) [13]. The binding energy of inhibitor N3 as predicted by AutoDock 4.2 was $-6.2 \mathrm{kcal} / \mathrm{mol}$ (Table 2). 

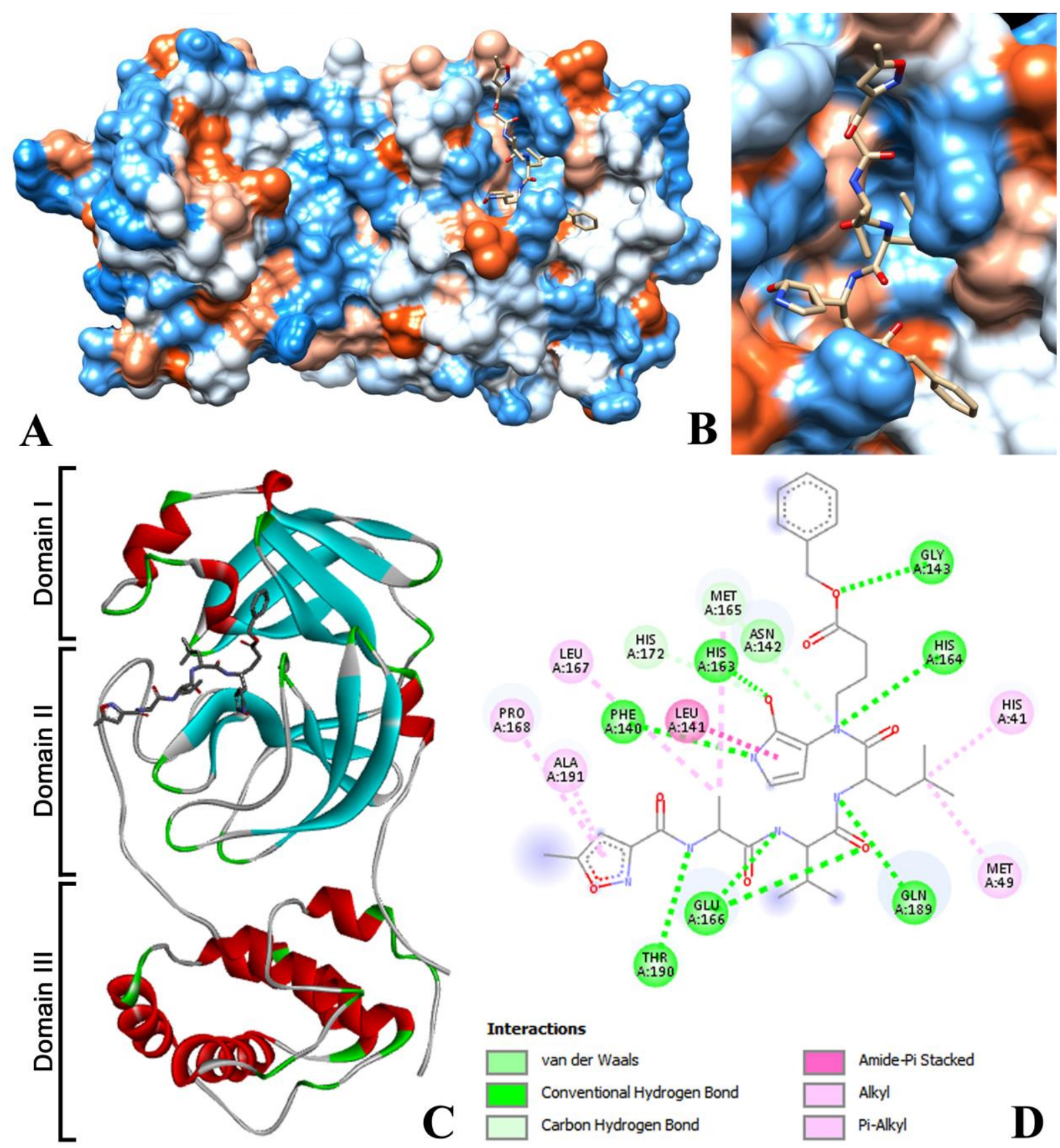

Figure 1. Interaction of $\mathrm{N} 3$ in the binding cleft of SARS-CoV-2 $\mathrm{M}^{\text {pro }}$ (A) hydrophobicity surface 3D representation (B) Interaction of $\mathrm{N} 3$ in a pocket site of SARS-CoV-2 $\mathrm{M}^{\text {pro }}(\mathrm{C})$ the crystal structure of SARS-CoV-2 $\mathrm{M}^{\text {pro }}$ in complex with an inhibitor N3 (D) 2D representation describing all possible bindings in active site of SARS-CoV-2 $\mathrm{M}^{\text {pro }}$

The drugs did not acquire more than 2 violations of Lipinski's rule were selected for molecular docking. The results of drug scanning using Lipinski's rule of five are shown in Table 1. These features are important for the identification of molecular drug ability. The detailed results on the binding energies and ZINC identification of each selected ligand are 
shown in Table 2. Lower binding energy values as well as maximum interactions with active site residues within the target $\mathrm{M}^{\text {pro }}$ of $6 \mathrm{LU} 7$ were evaluated for selecting the best pharmacophore-like drug-candidates. The lowest binding energies achieved from docking $6 \mathrm{LU} 7 \mathrm{M}^{\mathrm{pro}}$ with the ligands bergenin, dihydroartemisinin and quercitrin were $-7.93,-7.23$ and $-10.36 \mathrm{kcal} / \mathrm{mol}$, respectively. The mean binding energies achieved from docking 6LU7 $\mathrm{M}^{\text {pro }}$ with the ligands bergenin, dihydroartemisinin and quercitrin were $-7.86,-7.20$ and -10.29 $\mathrm{kcal} / \mathrm{mol}$, respectively (Table 2). The binding affinity seems higher as compared to inhibitor $\mathrm{N} 3(-6.2 \mathrm{kcal} / \mathrm{mol})$. The type and amount of bonding is essential for the high affinity of drug compounds with the active site of the protein. Bergenin forms many chemical bonds with seven active residues PHE140, GLY143, CYS145, HIS163, HIS164, GLU166 and GLN189 of 6LU7 $\mathrm{M}^{\text {pro }}$ receptor (Figure 2D). Dihydroartemisinin forms many chemical bonds with only three active residues GLY143, CYS145 and HIS163 of 6LU7 M ${ }^{\text {pro }}$ receptor (Figure 4D). Many chemical bonds were observed with only four active residues CYS145, HIS163, GLU166 and THR190 of 6LU7 $\mathrm{M}^{\text {pro }}$ receptor in case of quercitrin. However, several amino acids other than active residues were also bonded with $6 \mathrm{LU} 7 \mathrm{M}^{\text {pro }}$ receptor. Hence, binding affinity seems higher for quercitrin as compared to bergenin and dihydroartemisinin (Figure 3D). These findings ranked the inhibition potential of tested compounds as per binding energy in following order: quercitrin>bergenin>dihydroartemisinin. However, looking towards the number of active sites bonded, the rank of the inhibition potential of tested compounds is bergenin>quercitrin >dihydroartemisinin. 
Table 1. Properties of SARS-CoV-2 $\mathrm{M}^{\text {pro }}$ potential inhibitors

\begin{tabular}{|c|c|c|c|c|c|c|}
\hline \multirow[t]{2}{*}{ No } & \multirow[t]{2}{*}{ Compound } & \multirow[t]{2}{*}{ Structure } & \multirow[t]{2}{*}{ Source } & \multirow{2}{*}{$\begin{array}{c}\text { Molecular } \\
\text { formula }\end{array}$} & \multicolumn{2}{|c|}{ Lipinski's rule of five } \\
\hline & & & & & Properties & Value \\
\hline 1 & Bergenin & & Dictyophora indusiata & $\mathrm{C}_{14} \mathrm{H}_{16} \mathrm{O}_{9}$ & $\begin{array}{l}\text { Molecular weight }(<500 \mathrm{~g} / \mathrm{mol}) \\
\text { LogP }(<5) \\
\text { H-Bond donor }(<5) \\
\text { H-bond acceptor }(<10) \\
\text { Violations }\end{array}$ & $\begin{array}{l}328.27 \mathrm{~g} / \mathrm{mol} \\
-1.201 \\
5 \\
9 \\
0\end{array}$ \\
\hline 2 & Dihydroartemisinin & & Cyathus stercoreus & $\mathrm{C}_{15} \mathrm{H}_{24} \mathrm{O}_{5}$ & $\begin{array}{l}\text { Molecular weight }(<500 \mathrm{~g} / \mathrm{mol}) \\
\text { LogP }(<5) \\
\text { H-Bond donor }(<5) \\
\text { H-bond acceptor }(<10) \\
\text { Violations }\end{array}$ & $\begin{array}{l}284.35 \mathrm{~g} / \mathrm{mol} \\
2.5 \\
1 \\
5 \\
0\end{array}$ \\
\hline 3 & Quercitrin & & Geastrum triplex & $\mathrm{C}_{21} \mathrm{H}_{20} \mathrm{O}_{11}$ & $\begin{array}{l}\text { Molecular weight }(<500 \mathrm{~g} / \mathrm{mol} \\
\text { LogP }(<5) \\
\text { H-Bond donor }(<5) \\
\text { H-bond acceptor }(<10) \\
\text { Violations }\end{array}$ & $\begin{array}{l}448.38 \mathrm{~g} / \mathrm{mol} \\
0.9 \\
7 \\
11 \\
2\end{array}$ \\
\hline
\end{tabular}


Table 2. Molecular docking analysis of potential compounds against SARS-CoV-2 $\mathrm{M}^{\text {pro }}$

\begin{tabular}{lllcc}
\hline No. & Compound & Zinc/ Pubchem ID & $\begin{array}{l}\text { Lowest Binding } \\
\text { Energy }(\mathrm{kcal} / \mathrm{mol})\end{array}$ & $\begin{array}{l}\text { Mean Binding } \\
\text { Energy }(\mathrm{kcal} / \mathrm{mol})\end{array}$ \\
\hline 1 & Bergenin & ZINC4046820 & -7.93 & -7.86 \\
2 & Dihydroartemisinin & Pubchem CID_456410 & -7.23 & -7.20 \\
3 & Quercitrin & ZINC4175638 & -10.36 & -10.29 \\
4 & & N3- Peptide-like & -6.6 & -6.2 \\
\hline
\end{tabular}

The docking analysis of bergenin (Figure 2) indicated that active residue HIS163 exhibited conventional $\mathrm{H}$-bond, $\pi$-cation electrostatic interaction and $\pi$-hydrophobic and $\pi$ - $\pi$-T-shaped hydrophobic interactions with bergenin. Active residue GLN189 showed conventional Hbond, carbon-hydrogen bond and $\pi$-donor hydrogen bond interactions with bergenin. Conventional hydrogen bond interactions were also observed with an active residue of GLY143 and GLU166. Carbon-hydrogen bond and $\pi$-donor hydrogen bond interactions were found with PHE140 and HIS164 (Figure 5A). Active residue CYS145 formed $\pi$-alkyl hydrophobic interactions with bergenin (Figure 5B). Additionally, two more interactions, conventional H-bond and carbon-hydrogen interaction were observed with SER144 and HIS41, respectively.

The docking analysis of quercitrin is shown in Figure 3. The results indicated that conventional H-bond was observed with active residue HIS163, THR190 and with other amino acids TYR54 and GLN192. The active residue GLU166, THR190 and other amino acids PRO168, ARG188, HIS41 exhibited both carbon-hydrogen bond and $\pi$-donor hydrogen bond interactions with quercitrin (Figure 5C). Amino acids LEU 167 and MET165 formed alkyl hydrophobic interactions whereas amino acids MET165 and MET49 formed $\pi$-alkyl hydrophobic interactions with quercitrin (Figure 5D). Additionally, active residue CYS145 interacted by a sulphur bond with quercitrin.

The results of the docking analysis of dihydroartemisinin are shown in Figure 3. The results indicated that active residue CYS145 exhibited conventional H-bond and alkyl hydrophobic interactions with dihydroartemisinin (Figure 5E and 5F). Conventional H-bond and $\pi$-alkyl hydrophobic interactions were found with the active residues GLY143 and HIS163, respectively. Amino acid SER144, LEU141, MET49 and HIS41 exhibited conventional Hbond, carbon-hydrogen bond, alkyl hydrophobic interactions and $\pi$-alkyl hydrophobic interactions with dihydroartemisinin, respectively. 

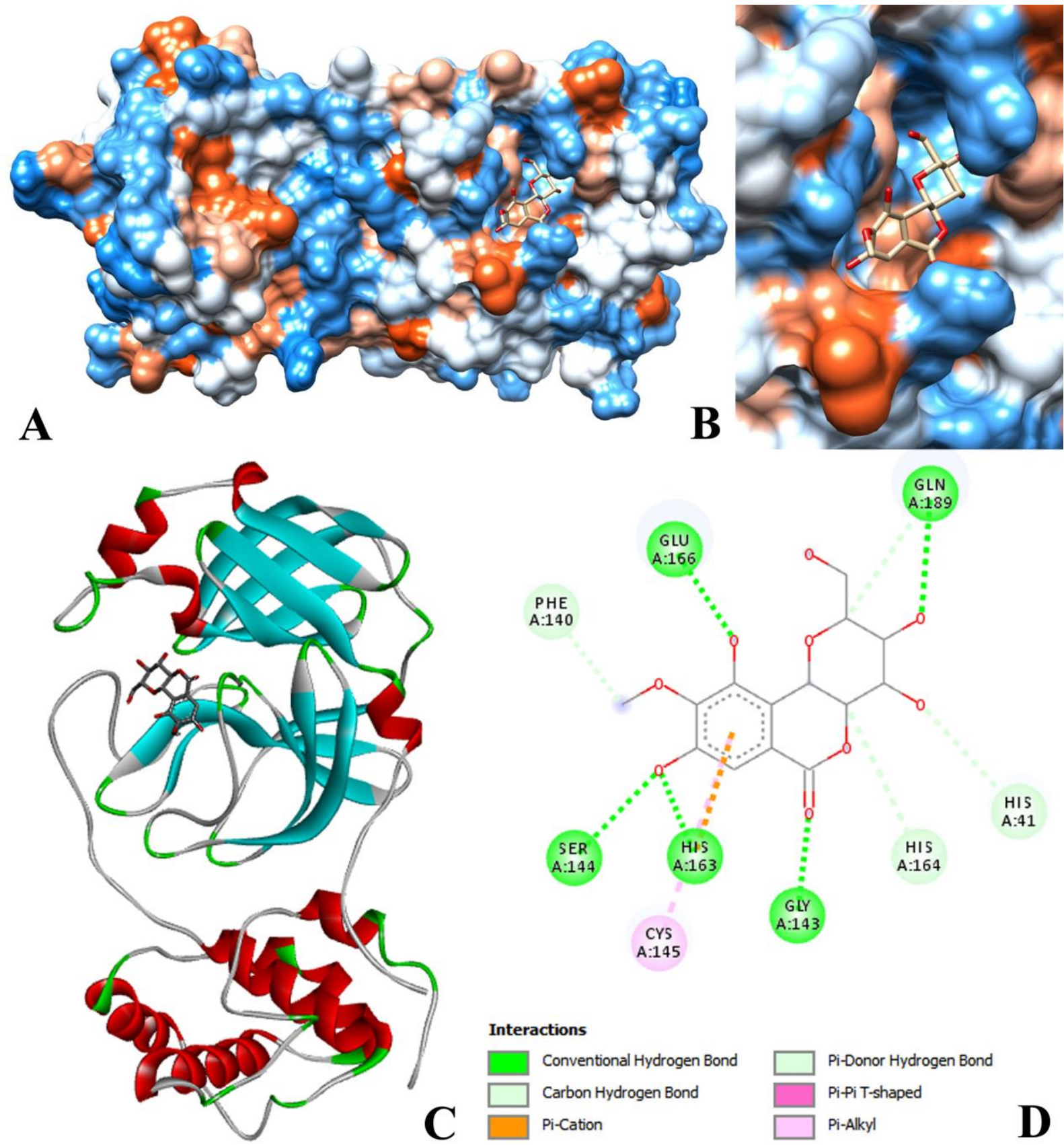

Figure 2. Docking analysis visualization of SARS-CoV-2 $\mathrm{M}^{\text {pro }}$ binding with Bergenin (A) hydrophobicity surface 3D representation (B) Interaction of Bergenin in a pocket site of SARS-CoV-2 $\mathrm{M}^{\text {pro }}$ (C) 3D representation of Bergenin-SARS-CoV-2 $\mathrm{M}^{\text {pro }}$ interaction (D) 2D representation describing bindings of Bergenin in active site of SARS-CoV-2 $\mathrm{M}^{\text {pro }}$ 

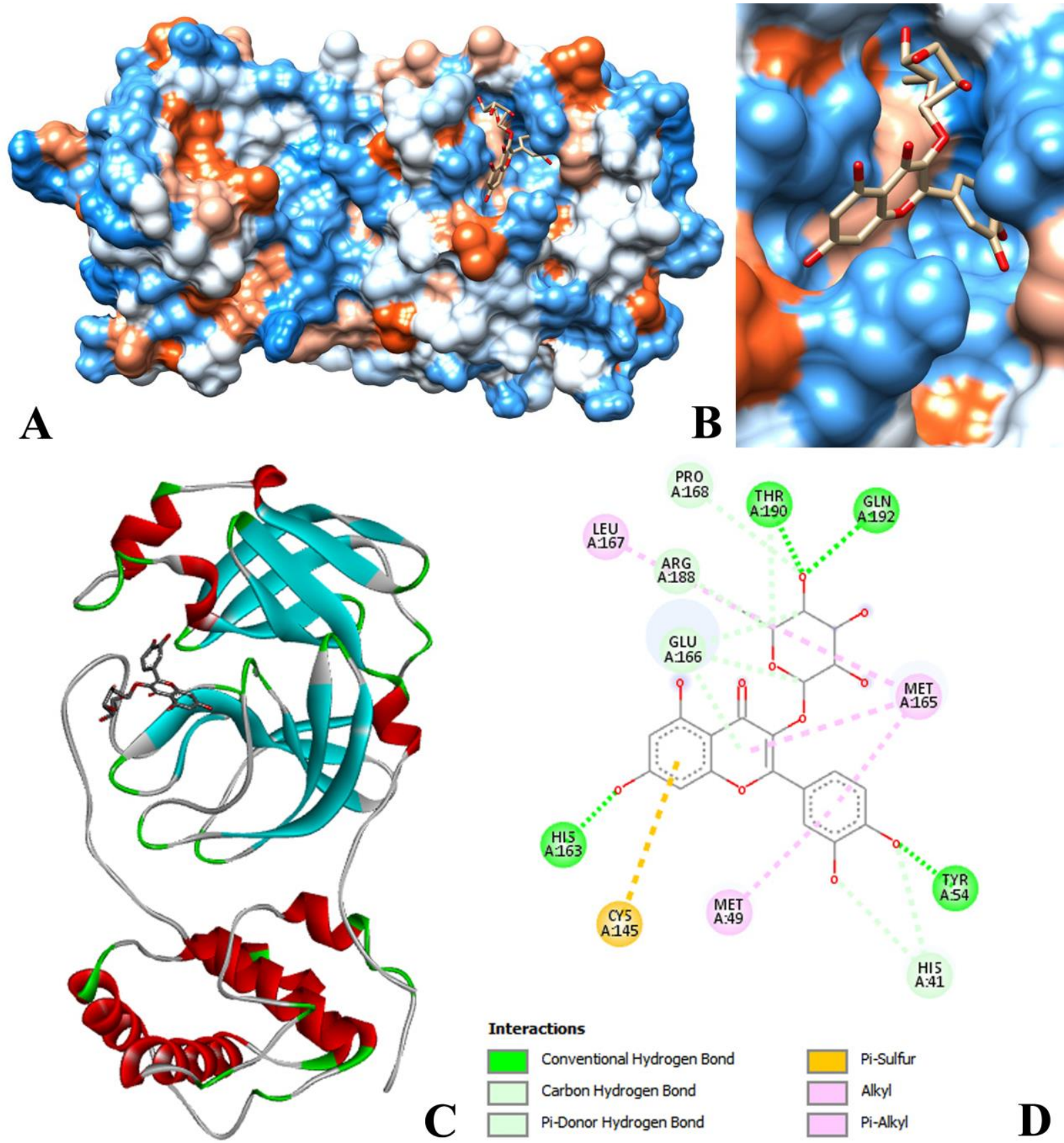

Figure 3. Docking analysis visualization of SARS-CoV-2 $\mathrm{M}^{\text {pro }}$ binding with Quercitrin (A) hydrophobicity surface 3D representation (B) Interaction of Quercitrin in a pocket site of SARS-CoV-2 $\mathrm{M}^{\text {pro }}$ (C) 3D representation of Quercitrin-SARS-CoV-2 $\mathrm{M}^{\text {pro }}$ interaction (D) 2D representation describing bindings of Quercitrin in active site of SARS-CoV-2 $\mathrm{M}^{\text {pro }}$ 

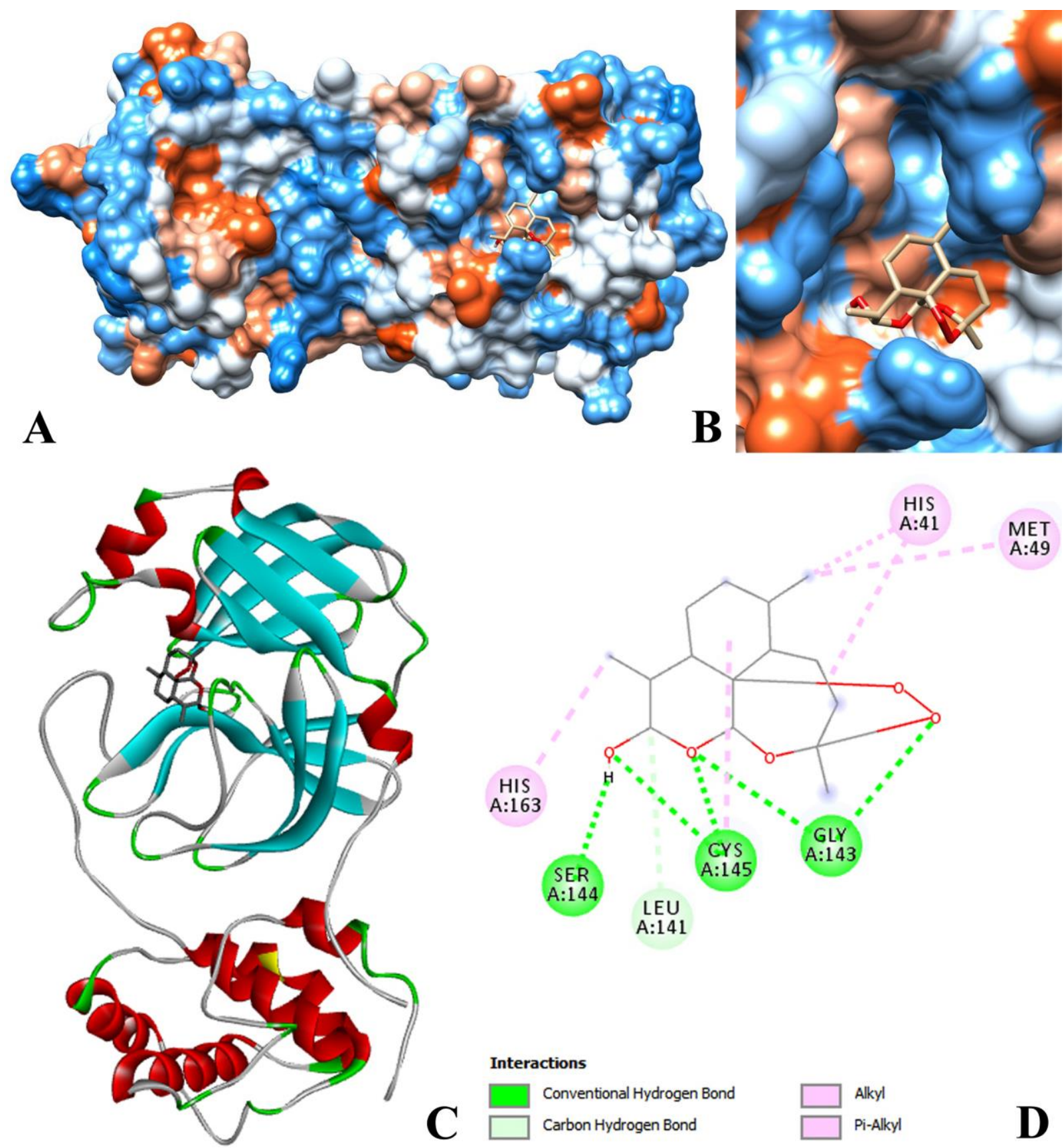

D

Figure 4. Docking analysis visualization of SARS-CoV-2 $\mathrm{M}^{\text {pro }}$ binding with Dihydroartemisinin (A) hydrophobicity surface 3D representation (B) Interaction of Dihydroartemisinin in a pocket site of SARS-CoV-2 $\mathrm{M}^{\text {pro }}$ (C) 3D representation of Dihydroartemisinin-SARS-CoV-2 $\mathrm{M}^{\text {pro }}$ interaction (D) 2D representation describing bindings of Dihydroartemisinin in active site of SARS-CoV-2 $\mathrm{M}^{\text {pro }}$ 

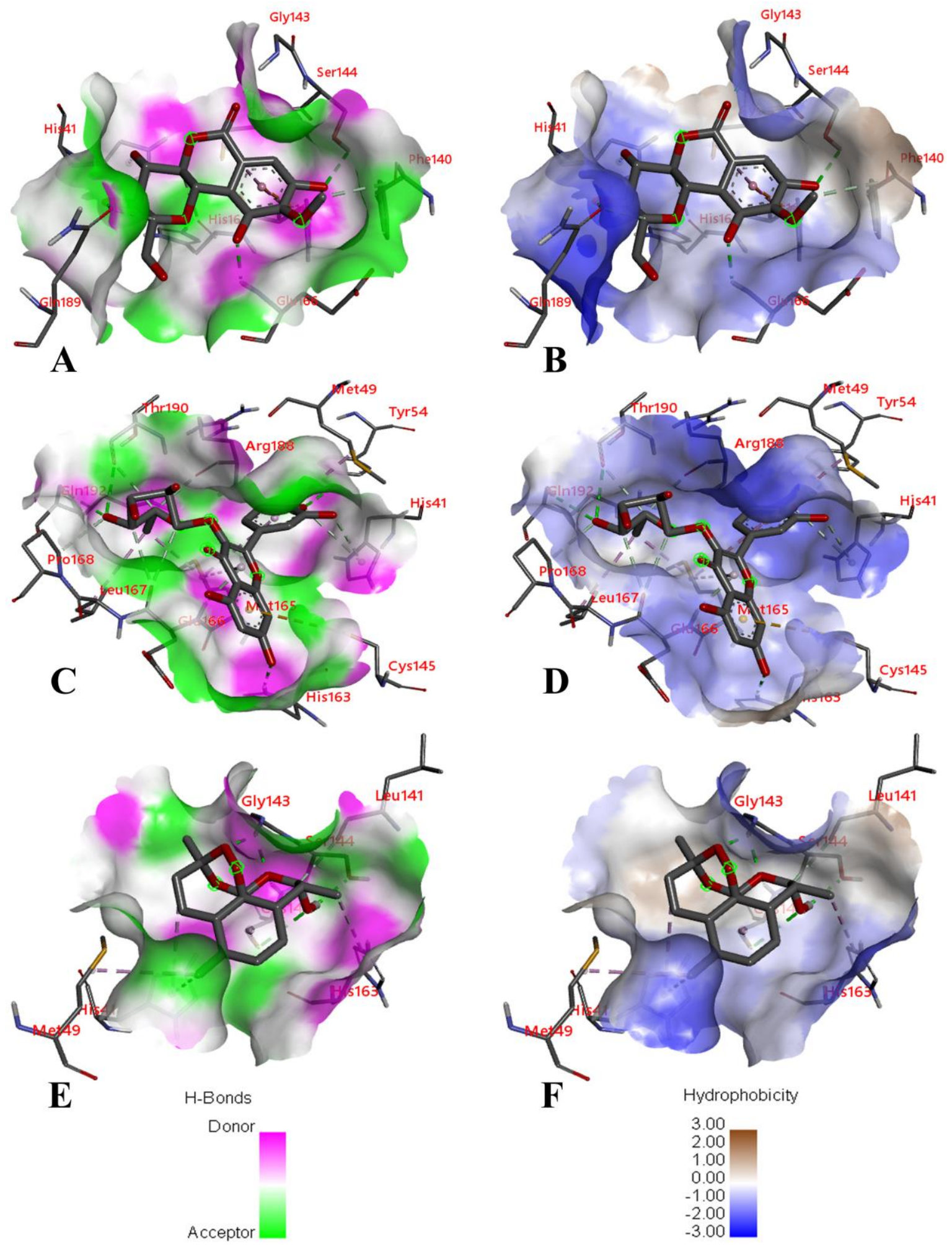

Figure 5. Interactions of drugs with H-bond and hydrophobic in a pocket site of SARS-CoV$2 \mathrm{M}^{\text {pro }}(\mathrm{A})$, (C) and (E) show H-bond interaction of Bergenin, Quercitrin and Dihydroartemisinin; (B), (D) and (F) show hydrophobic interaction of Bergenin, Quercitrin and Dihydroartemisinin 


\subsection{ADMET study}

Table 3. ADMET prediction of the bergenin, quercitrin and dihydroartemisinin

\begin{tabular}{|c|c|c|c|}
\hline & Bergenin & Quercitrin & Dihydroartemisinin \\
\hline \multicolumn{4}{|l|}{ Absorption } \\
\hline Water solubility $(\log \mathrm{mol} / \mathrm{L})$ & -2.068 & -3.006 & -3.124 \\
\hline $\mathrm{CaCO}_{2}$ permeability (log Papp in $10^{-6} \mathrm{~cm} / \mathrm{s}$ ) & 0.363 & -0.576 & 1.228 \\
\hline Intestinal absorption (\% Absorbed) & 34.88 & 50.59 & 95.071 \\
\hline Skin Permeability (log Kp) & -2.735 & -2.735 & -3.764 \\
\hline P-glycoprotein substrate & Yes & Yes & No \\
\hline P-glycoprotein I inhibitor & No & No & No \\
\hline P-glycoprotein II inhibitor & No & No & No \\
\hline \multicolumn{4}{|l|}{ Distribution } \\
\hline VDss (human) (log L/kg) & 0.946 & -0.262 & 0.503 \\
\hline Fraction unbound (human) (Fu) & 0.562 & 0.182 & 0.438 \\
\hline BBB permeability $(\log \mathrm{BB})$ & -1.334 & -1.683 & 0.393 \\
\hline CNS permeability (log PS) & -4.267 & -4.708 & -2.901 \\
\hline \multicolumn{4}{|l|}{ Metabolism } \\
\hline CYP2D6 substrate & No & No & No \\
\hline CYP3A4 substrate & No & No & No \\
\hline CYP1A2 inhibitor & No & No & No \\
\hline CYP2C19 inhibitor & No & No & No \\
\hline CYP2C9 inhibitor & No & No & No \\
\hline CYP2D6 inhibitor & No & No & No \\
\hline CYP3A4 inhibitor & No & No & No \\
\hline \multicolumn{4}{|l|}{ Excretion } \\
\hline Total Clearance $(\log \mathrm{ml} / \mathrm{min} / \mathrm{kg})$ & 0.5 & 0.624 & 0.99 \\
\hline Renal OCT2 substrate & No & No & No \\
\hline \multicolumn{4}{|l|}{ Toxicity } \\
\hline AMES toxicity & No & No & No \\
\hline Max. tolerated dose (human) (log mg/kg/day) & -0.122 & 0.426 & -0.371 \\
\hline hERG I inhibitor & No & No & No \\
\hline hERG II inhibitor & No & Yes & No \\
\hline Oral Rat Acute Toxicity (LD50) (mol/kg) & 2.387 & 2.662 & 2.781 \\
\hline $\begin{array}{l}\text { Oral Rat Chronic Toxicity (LOAEL) (log } \\
\text { mg/kg_bw/day) }\end{array}$ & 3.868 & 3.762 & 1.925 \\
\hline Hepatotoxicity & No & No & No \\
\hline Skin Sensitisation & No & No & No \\
\hline Tetrahymena pyriformis toxicity $(\mu \mathrm{g} / \mathrm{L})$ & 0.285 & 0.285 & 0.306 \\
\hline Minnow toxicity $(\mathrm{mM})$ & 4.207 & 1.828 & 1.73 \\
\hline
\end{tabular}

ADMET predictions of the bergenin, quercitrin and dihydroartemisinin were carried out using the pkCSM - pharmacokinetics server which is shown in Table 3. Based on VDss data, bergenin showed better disdtibution followed by dihydroartemisinin and quercitrin. The results of fraction unbound also showed the abilities of these three drugs for distribution in 
tissues of body more effectively. All the three drugs did not interfere in the cytochrome (CYP) metabolism and have better renal clearance. The renal clearance was found much better in dihydroartemisinin followed by quercitrin and bergenin. Moreover, hepatotoxicity and skin sensitisation were also not predicted by all three drugs. The results of ADMET study further indicate the druggability potential of bergenin, quercitrin and dihydroartemisinin.

Reflecting back in the history, bergenin, dihydroartemisinin and quercitrin have been reported for various medicinal uses. Bergenin is a C-glucoside of 4-O-methyl gallic acid and has long been used as folk medicine in many parts of Asia [35]. It is poorly soluble in water and is easily degraded in a basic solution [36]. The molecular formula and chemical structure are shown in Table 1. Bergenin exhibits antiparasitic, antihepatotoxic, antiviral, anti-HIV, antiarrhythmic, antiulcerogenic, neuroprotective, immunomodulatory, antioxidant, antimicrobial and anti-inflammatory properties [37]. Anti-HIV activity of bergenin was reported with an effective concentration $\left(\mathrm{EC}_{50}\right)$ of $40 \mu \mathrm{g} / \mathrm{mL}$ [38]. Bergenin was also displayed antiviral activity against Hepatitis $\mathrm{C}$ virus with an $\mathrm{IC}_{50}$ of $1.71 \mathrm{mM}$ [39]. Antiviral activity of bergenin was also reported against herpes simplex virus type-1 with an $\mathrm{IC}_{50}$ value of $<6.25 \mu \mathrm{g} / \mathrm{mL}$ [40]. Additionally, Bergenin was also reported for a good antiparasitic activity with an $\mathrm{IC}_{50}$ value of $2.4 \mu \mathrm{g} / \mathrm{mL}$ against Plasmodium falciparum, a chloroquine sensitive strain [41].

Quercitrin is a glycoside made from the quercetin flavonoid and the deoxy sugar rhamnose. It was reported to show antiviral activity against dengue virus infection with an $\mathrm{IC}_{50}$ value of $467.27 \mu \mathrm{g} / \mathrm{mL}$ [42]. Quercitrin displayed inhibition against HIV-1 reverse transcriptase [43] and antiviral activity against hepatitis C virus (HCV) [44]. Quercitrin also showed proliferation inhibition of Plasmodium falciparum with an $\mathrm{IC}_{50}$ value of $1.1 \mu \mathrm{g} / \mathrm{mL}$ [45]. It also exhibited antimicrobial, antidiarrhoeic, antileishmanial and anti-inflammatory properties [46-49]. Above findings indicated that bergenin and quercitrin could be used against SARSCoV-2 as it has good antiviral activity against hepatitis and HIV viruses similar to remdesivir as well as antiparasitic activity similar to chloroquine, drugs which are being used currently for treatment of SARS-CoV-2 [19].

Dihydroartemisinin, an artemisinin derivative, is more water-soluble and is a safe and most effective antimalarial drug. Dihydroartemisinin is generally used with a combination of 
piperaquine, a popular regimen recommended by WHO against malaria. The dihydroartemisinin was reported to show $50 \%$ inhibition $\left(\mathrm{IC}_{50}\right)$ of Plasmodium falciparum growth in vitro in the low nanomolar range [50]. As shown in Fig. 4, dihydroartemisinin binds with the pocket region of active sites of $6 \mathrm{LU} 7 \mathrm{M}^{\text {pro }}$; therefore, it may probable potential molecule against SARS-CoV-2 and needs further studies to confirm the potential of drug.

The docking analysis, ADMET predictions and medicinal properties clearly indicated that bergenin, quercitrin and dihydroartemisinin were the most significant compounds as potential inhibitors of SARS-CoV-2 $\mathrm{M}^{\text {pro }}$, which could be explored further.

\section{Conclusions}

So far, there is no specified vaccine or drug that is available against SARS-CoV-2. To control this pandemic, it is necessary to develop specific novel inhibitors which can block the viral entry and its replication within the host cells. Many clinical trials related to vaccine and new drugs development against SARS-CoV-2 are on track. At present, computational approaches could help and lead scientific world in designing novel drugs against SARS-CoV-2 from different natural resources. On this line, fungi are an important source of the natural bioactive compounds that have potential as protease inhibitors and immunomodulators. In the present study, three compounds were identified from fungi as potent inhibitors of SARS-CoV-2 $\mathrm{M}^{\text {pro }}$ in an in-silico analysis which could be used for the prevention and treatment of SARS-CoV2. These molecules are already in use against various viral diseases and are of plant origin. Therefore, we recommend these compounds to combat SARS-CoV-2 infection. Laboratory assays and clinical tests are needed to fully understand the level of toxicity and pharmacokinetic profile due to their fungal origin. To the best of our knowledge, present research reports these three bioactive compounds from Dictyophora indusiata, Geassstrum triplex and Cyathus stercoreus for the first time.

\section{Authors contribution:}

RSP: Collection, identification of fungi, extraction of bioactive compound, analysis of HRLC/MS data, compound screening, molecular docking result analysis, manuscript preparation and compilation of results from other collaborators. AGV: Screening of bioactive compound for docking analysis, review literature, molecular docking, interpretation of the results and manuscript preparation. NRP: Screening of bioactive compound and molecules for docking analysis using different software, interpretation of docking result and writing the MS. AMV: 
Collection and identification of fungi, review literature, writing MS. SMP: sample processing for extraction of compounds, technical support for work, interpretation and writing and, KSR: Conceived the concept, collection and identification of fungi, field works, laboratory and administrative support with final editing and improving the manuscript.

\section{Acknowledgments}

Authors are greatly thankful to Mr. Mayank Tandel, Mr. Kaushik Tailor and Mr. Ronak Kachhiyapatel for their necessary assistance during the laboratory work and analysis of results of the present research work.

\section{References}

[1] J. Cui, F. Li, Z.-L. Shi, Origin and evolution of pathogenic coronaviruses, Nat. Rev. Microbiol. 17 (2019) 181-192.

[2] D. Schoeman, B.C. Fielding, Coronavirus envelope protein: current knowledge, Virol. J. 16 (2019) 69.

[3] J.S.M. Peiris, Y. Guan, K.Y. Yuen, Severe acute respiratory syndrome, Nat. Med. 10 (2004) S88-S97.

[4] A.M. Zaki, S. Van Boheemen, T.M. Bestebroer, A.D.M.E. Osterhaus, R.A.M. Fouchier, Isolation of a novel coronavirus from a man with pneumonia in Saudi Arabia, N. Engl. J. Med. 367 (2012) 1814-1820.

[5] D. Benvenuto, M. Giovanetti, A. Ciccozzi, S. Spoto, S. Angeletti, M. Ciccozzi, The 2019-new coronavirus epidemic: evidence for virus evolution, J. Med. Virol. 92 (2020) $455-459$.

[6] F. Wu, S. Zhao, B. Yu, Y.-M. Chen, W. Wang, Z.-G. Song, Y. Hu, Z.-W. Tao, J.-H. Tian, Y.-Y. Pei, A new coronavirus associated with human respiratory disease in China, Nature. 579 (2020) 265-269.

[7] M. Cascella, M. Rajnik, A. Cuomo, S.C. Dulebohn, R. Di Napoli, Features, evaluation and treatment coronavirus (COVID-19), in: Statpearls [Internet], StatPearls Publishing, 2020.

[8] M. Zheng, Y. Gao, G. Wang, G. Song, S. Liu, D. Sun, Y. Xu, Z. Tian, Functional exhaustion of antiviral lymphocytes in COVID-19 patients, Cell. Mol. Immunol. (2020) 1-3.

[9] L. Zhang, D. Lin, X. Sun, U. Curth, C. Drosten, L. Sauerhering, S. Becker, K. Rox, R. 
Hilgenfeld, Crystal structure of SARS-CoV-2 main protease provides a basis for design of improved $\alpha$-ketoamide inhibitors, Science (80-. ). 368 (2020) 409-412.

[10] COVID-19 CORONAVIRUS PANDEMIC, https://www.worldometers.info/coronavirus/ (accessed June 19, 2020).

[11] S.P. Gupta, Viral proteases and their inhibitors, Academic Press, 2017.

[12] K. Anand, G.J. Palm, J.R. Mesters, S.G. Siddell, J. Ziebuhr, R. Hilgenfeld, Structure of coronavirus main proteinase reveals combination of a chymotrypsin fold with an extra $\alpha$-helical domain, EMBO J. 21 (2002) 3213-3224.

[13] Z. Jin, X. Du, Y. Xu, Y. Deng, M. Liu, Y. Zhao, B. Zhang, X. Li, L. Zhang, C. Peng, Structure of M pro from SARS-CoV-2 and discovery of its inhibitors, Nature. (2020) $1-5$.

[14] X. Liu, B. Zhang, Z. Jin, H. Yang, Z. Rao, The crytal structure of 2019-nCoV main protease in complex with an inhibitor N3, RCSB Protein Data Bank. (2020).

[15] X. Xu, P. Chen, J. Wang, J. Feng, H. Zhou, X. Li, W. Zhong, P. Hao, Evolution of the novel coronavirus from the ongoing Wuhan outbreak and modeling of its spike protein for risk of human transmission, Sci. China Life Sci. 63 (2020) 457-460.

[16] P. Colson, J.-M. Rolain, D. Raoult, Chloroquine for the 2019 novel coronavirus, Int J Antimicrob Agents. 55 (2020) 105923.

[17] Z. Sahraei, M. Shabani, S. Shokouhi, A. Saffaei, Aminoquinolines against coronavirus disease 2019 (COVID-19): chloroquine or hydroxychloroquine, Int J Antimicrob Agents. 105945 (2020).

[18] M. Wang, R. Cao, L. Zhang, X. Yang, J. Liu, M. Xu, Z. Shi, Z. Hu, W. Zhong, G. Xiao, Remdesivir and chloroquine effectively inhibit the recently emerged novel coronavirus (2019-nCoV) in vitro, Cell Res. 30 (2020) 269-271.

[19] T.P. Sheahan, A.C. Sims, S.R. Leist, A. Schäfer, J. Won, A.J. Brown, S.A. Montgomery, A. Hogg, D. Babusis, M.O. Clarke, Comparative therapeutic efficacy of remdesivir and combination lopinavir, ritonavir, and interferon beta against MERSCoV, Nat. Commun. 11 (2020) 1-14.

[20] N. Suwannarach, J. Kumla, K. Sujarit, T. Pattananandecha, C. Saenjum, S. Lumyong, Natural Bioactive Compounds from Fungi as Potential Candidates for Protease Inhibitors and Immunomodulators to Apply for Coronaviruses, Molecules. 25 (2020) 1800.

[21] A. Fredenhagen, F. Petersen, M. Tintelnot-Blomley, J. RÖSEL, H. Mett, P. Hug, 
Semicochliodinol A and B: inhibitors of HIV-1 protease and EGF-R protein tyrosine kinase related to asterriquinones produced by the fungus Chrysosporium merdarium, J. Antibiot. (Tokyo). 50 (1997) 395-401.

[22] C. Sillapachaiyaporn, S. Nilkhet, A.T. Ung, S. Chuchawankul, Anti-HIV-1 protease activity of the crude extracts and isolated compounds from Auricularia polytricha, BMC Complement. Altern. Med. 19 (2019) 1-10.

[23] U.W. Hawas, S. El-Desouky, L.A. El-Kassem, W. Elkhateeb, Alternariol derivatives from Alternaria alternata, an endophytic fungus residing in red sea soft coral, inhibit HCV NS3/4A protease, Appl. Biochem. Microbiol. 51 (2015) 579-584.

[24] L.-Y. Yang, J. Lin, B. Zhou, Y.-G. LIU, B.-Q. ZHU, H1-A, a compound isolated from Fusarium oxysporum inhibits hepatitis C virus (HCV) NS3 serine protease, Chin. J. Nat. Med. 14 (2016) 299-302.

[25] P. Rao, A. Shukla, P. Parmar, D. Goswami, Proposing a fungal metabolite-Flaviolin as a potential inhibitor of 3CLpro of novel coronavirus SARS-CoV2 using docking and molecular dynamics, ArXiv Prepr. ArXiv2004.03806. (2020).

[26] S. Khaerunnisa, H. Kurniawan, R. Awaluddin, S. Suhartati, S. Soetjipto, Potential inhibitor of COVID-19 main protease (Mpro) from several medicinal plant compounds by molecular docking study, Prepr. Doi10. 20944/Preprints202003. 0226. V1. (2020) $1-14$.

[27] ZINC database, https://zinc15.docking.org/ (accessed April 28, 2020).

[28] PubChem, https://pubchem.ncbi.nlm.nih.gov/ (accessed April 28, 2020).

[29] C.A. Lipinski, F. Lombardo, B.W. Dominy, P.J. Feeney, Experimental and computational approaches to estimate solubility and permeability in drug discovery and development settings, Adv. Drug Deliv. Rev. 64 (2012) 4-17.

[30] SWISSADME, http://www.swissadme.ch/ (accessed April 28, 2020).

[31] B. Andrade, P. Ghosh, D. Barth, S. Tiwari, R.J. Santana, W.R. de A.S. Silva, T.S. Melo, A. dos Santos Freitas, P. González-Grande, L.S. Palmeira, Computational screening for potential drug candidates against SARS-CoV-2 main protease, (2020).

[32] G.M. Morris, R. Huey, W. Lindstrom, M.F. Sanner, R.K. Belew, D.S. Goodsell, A.J. Olson, AutoDock4 and AutoDockTools4: Automated docking with selective receptor flexibility, J. Comput. Chem. 30 (2009) 2785-2791.

[33] Schrödinger, The PyMOL Molecular Graphics System, Version 2.3, (2020).

[34] D.E. V Pires, T.L. Blundell, D.B. Ascher, pkCSM: predicting small-molecule 
pharmacokinetic and toxicity properties using graph-based signatures, J. Med. Chem. 58 (2015) 4066-4072.

[35] D.K. Patel, K. Patel, R. Kumar, M. Gadewar, V. Tahilyani, Pharmacological and analytical aspects of bergenin: a concise report, Asian Pacific J. Trop. Dis. 2 (2012) $163-167$.

[36] G.B. Bajracharya, Diversity, pharmacology and synthesis of bergenin and its derivatives: potential materials for therapeutic usages, Fitoterapia. 101 (2015) 133152.

[37] U. Singh, A. Barik, K.I. Priyadarsini, Reactions of hydroxyl radical with bergenin, a natural poly phenol studied by pulse radiolysis, Bioorg. Med. Chem. 17 (2009) 60086014.

[38] S. Piacente, C. Pizza, N. De Tommasi, N. Mahmood, Constituents of Ardisia japonica and their in vitro anti-HIV activity, J. Nat. Prod. 59 (1996) 565-569.

[39] G.-Y. Zuo, Z.-Q. Li, L.-R. Chen, X.-J. Xu, In vitro anti-HCV activities of Saxifraga melanocentra and its related polyphenolic compounds, Antivir. Chem. Chemother. 16 (2005) 393-398.

[40] M. Rajbhandari, M. Lalk, R. Mentel, U. Lindequist, Antiviral Activity and Constituents of the Nepalese Medicinal Plant Astilbe rivularis, Rec. Nat. Prod. 5 (2011).

[41] G. Uddin, A. Sadat, B.S. Siddiqui, Comparative Antioxidant and Antiplasmodial Activities of 11-O-Galloylbergenin and Bergenin Isolated from Bergenia ligulata, World Appl. Sci. J. 27 (2013) 977-981.

[42] K.H. Chiow, M.C. Phoon, T. Putti, B.K.H. Tan, V.T. Chow, Evaluation of antiviral activities of Houttuynia cordata Thunb. extract, quercetin, quercetrin and cinanserin on murine coronavirus and dengue virus infection, Asian Pac. J. Trop. Med. 9 (2016) 1-7.

[43] Y.-B. Yu, H. Miyashiro, N. Nakamura, M. Hattori, J.C. Park, Effects of triterpenoids and flavonoids isolated from Alnus firma on HIV-1 viral enzymes, Arch. Pharm. Res. 30 (2007) 820 .

[44] C. Aoki, S.R.I. Hartati, M.R. Santi, R. Firdaus, M. Hanafi, L.B.S. Kardono, Y. Shimizu, P.P. Sudarmono, H. Hotta, Isolation and identification of substances with anti-hepatitis C virus activities from Kalanchoe pinnata, Int. J. Pharm. Pharm. Sci. 6 (2014) 211-215.

[45] Y. Liu, N. Murakami, H. Ji, P. Abreu, S. Zhang, Antimalarial Flavonol Glycosides 
from Euphorbia hirta, Pharm. Biol. 45 (2007) 278-281.

[46] M. Comalada, D. Camuesco, S. Sierra, I. Ballester, J. Xaus, J. Gálvez, A. Zarzuelo, In vivo quercitrin anti-inflammatory effect involves release of quercetin, which inhibits

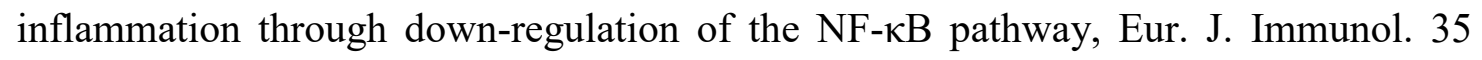
(2005) 584-592.

[47] M.F. Muzitano, L.W. Tinoco, C. Guette, C.R. Kaiser, B. Rossi-Bergmann, S.S. Costa, The antileishmanial activity assessment of unusual flavonoids from Kalanchoe pinnata, Phytochemistry. 67 (2006) 2071-2077.

[48] S. Hasan, K. Singh, M. Danisuddin, P.K. Verma, A.U. Khan, Inhibition of major virulence pathways of Streptococcus mutans by quercitrin and deoxynojirimycin: a synergistic approach of infection control, PLoS One. 9 (2014).

[49] J. Galvez, M.E. Crespo, J. Jimenez, A. Suarez, A. Zarzuelo, Antidiarrhoeic activity of quercitrin in mice and rats, J. Pharm. Pharmacol. 45 (1993) 157-159.

[50] G.M. Keating, Dihydroartemisinin/Piperaquine, Drugs. 72 (2012) 937-961. 